\title{
APLICABILIDADE DO MÉTODO DE SIMULAÇÃO DE MONTE CARLO NA PREVISÃO DOS CUSTOS DE PRODUÇÃO DE COMPANHIAS INDUSTRIAIS: O CASO DA COMPANHIA VALE DO RIO DOCE
}

\section{APPLYING MONTE CARLO SIMULATION IN PREDICTING COSTS OF MANUFACTURING COMPANIES: THE CASE OF COMPANHIA VALE DO RIO DOCE}

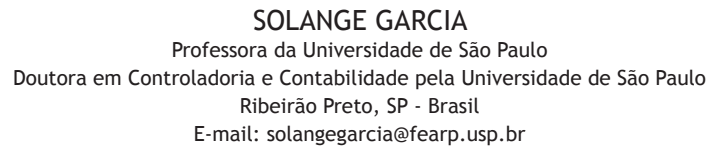

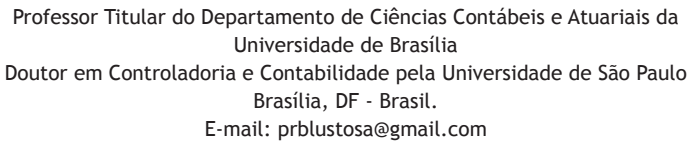

NARA ROSA BARROS

Graduada em Ciências Contábeis pelo Departamento de Contabilidade da Universidade de Brasília

E-mail: naraunb@gmail.com

\section{RESUMO}

A necessidade de decisões qualificadas no ambiente empresarial gera demandas pelo uso de métodos matemáticos e estatísticos no auxílio ao processo decisório. Nesse contexto, o presente artigo objetiva testar a aplicabilidade do Método de Simulação de Monte Carlo para prever variações nos custos de produção em um período pós-privatização. Para realizar o experimento foi escolhida a Companhia Vale do Rio Doce - CVRD, considerando a sua privatização ocorrida em 1997. Os dados das variáveis analisadas foram extraídos das demonstrações contábeis publicadas entre 1990 e 2004. Os custos de produção (CPV) são simulados em duas aplicações do Método: aplicação que desconsidera os efeitos da privatização e aplicação que considera, no período pós privatização, a redução de custos variáveis e o incremento de receitas operacionais. Em um processo de privatização, esses efeitos são esperados e foram estimados por meio de regressões lineares em estudo anterior de Oliveira e Lustosa (2007). Para verificar a capacidade preditiva do método são realizadas análises comparativas, por meio de testes estatísticos de médias, entre as amostras reais e as amostras simuladas do período pós-privatização. Os resultados obtidos evidenciam a adequação do Método na previsão dos custos de produção e consequente auxílio ao processo decisório.

Palavras-chave: Método de Simulação de Monte Carlo; Custos de Produção; Privatização; Companhia Vale do Rio Doce.

\section{ABSTRACT}

The need for qualified decisions in business environment creates demands for the use of mathematical and statistical methods to assist the decisional process. In this context, this article aims to test the applicability of the method of Monte Carlo simulation to predict changes in production costs in a post-privatization period. To perform the experiment Companhia Vale do Rio Doce - CVRD was chosen, considering its privatization that occurred in 1997. The analyzed variables data were extracted from published financial statements between 1990 and 2004. Production costs (CPV) are simulated in two applications of the method: an application that ignores the effects of privatization and implementation that considers the period after privatization, the reduction of variable costs and the income operating increase. In a privatization process, these effects are expected and were estimated by linear regressions in a previous study by Lustosa and Oliveira (2007). To verify the predictive ability of the method comparative analysis are performed, using statistical tests of means between the real samples and the post-privatization period simulated samples. The results show the suitability of the method in predicting the production costs and consequently assisting the decisional process.

Key-words: Method of Monte Carlo Simulation. Predicting Costs of Industrial Production. 


\section{INTRODUÇÃO}

No âmbito da redução da participação estatal na economia brasileira nas décadas de 80 e 90, diversos pesquisadores [OLIVEIRA; LUSTOSA, 2007; COLODETI FILHO; GOMES; TEIXEIRA, 2003; CHAN; SANTOS; SILVA, 2003; OLIVEIRA; LIMA, 2002] focaram seus estudos na análise das companhias privatizadas, tanto na esfera operacional quanto financeira. Esses estudos incentivaram a busca por evidências de melhoria produtiva.

Com o crescimento da complexidade dos problemas reais e a evolução dos sistemas computacionais, a simulação aparece como um instrumento cada vez mais utilizado nas mais variadas áreas de conhecimento. Nesse contexto, a simulação é utilizada para o estudo de problemas, geralmente complexos, para os quais não se dispõe de solução analítica. Em termos mais práticos, consiste no desenvolvimento de um modelo ou representação de uma situação real (ou ainda por existir) e, por intermédio do uso do computador, possibilita a realização de experimentos com vários cenários. Desse modo, é uma ferramenta de auxílio na avaliação de sistemas fornecendo uma melhor compreensão ao invés de gerar simplesmente uma solução. A Simulação de Monte Carlo é utilizada na avaliação de fenômenos que se podem caracterizar por um comportamento probabilístico. Por meio da geração de números aleatórios, permite resolver uma quantidade grande de problemas com a simulação de cenários e o posterior cálculo de um valor esperado. Esse método admite a implantação de hipóteses adicionais nas previsões.

Em um processo de privatização a expectativa é de melhoria na eficiência operacional, por meio de economias de custo, racionalização de processos e eliminação de desperdícios. Aspecto que é ressaltado em quase todos os trabalhos que tratam do processo de privatização brasileiro, sendo exemplos, Rosa e Hoffman (1995), Sampaio, Ramos e Sampaio (2005) e Anuatti-Neto et al (2005).

Assim, Oliveira e Lustosa (2007) testam a hipótese de melhoria da eficiência produtiva da Companhia Vale do Rio Doce - CVRD a partir da identificação empírica do comportamento dos seus custos de fabricação. Para tanto foi necessária a identificação dos componentes fixos e variáveis envolvidos no processo produtivo por meio da análise de regressão linear. Os autores utilizaram a inferência de que o custo dos produtos vendidos - CPV é uma função linear da receita operacional líquida - ROL e os parâmetros da regressão representam os custos fixos e os custos variáveis da Companhia. Os resultados demonstram que houve uma alteração significativa no fator de custo variável unitário, o qual passou de 0,8036 a 0,5399, representando aproximadamente $33 \%$ de redução de custos no período pós-privatização. Também foi verificado o incremento de receitas operacionais neste período.

Nesse sentido, o objetivo deste trabalho é testar a aplicabilidade do Método de Simulação de Monte Carlo para prever variações nos custos de produção após a desestatização da CVRD, considerando os impactos desse fator. Para tanto, os dados simulados encontrados serão comparados, por meios estatísticos, com os dados reais e confrontados com a pesquisa bibliográfica. Com isso, pretende-se responder a 
seguinte questão de pesquisa: O modelo de Simulação de Monte Carlo é viável como metodologia para previsão dos custos de produção da empresa analisada?

0 presente estudo mostra-se relevante devido à escassez de pesquisas que buscam analisar a aplicabilidade de métodos matemáticos no auxílio aos gestores quando do processo decisório, sendo esse um campo ainda a se explorar no Brasil.

O trabalho está dividido em cinco sessões. Após essa introdução, é apresentado o referencial teórico. A terceira seção proporciona uma visualização do desenho da pesquisa, indicando como foi realizada, a fonte de informações e seu tratamento. A seção subsequente evidencia os resultados obtidos com a pesquisa, bem como os comentários pertinentes. Por último, a quinta sessão apresenta as considerações finais.

\section{REFERENCIAL TEÓRICO}

\subsection{A PRIVATIZAÇÃO NO BRASIL E OS SEUS IMPACTOS NA EFICIÊNCIA OPERACIONAL}

O processo de privatização que ocorreu no Brasil na década de 1990 foi estimulado pela existência de incentivos tanto pelo lado do Estado brasileiro, proprietário das empresas, como pelo lado dos compradores privados. Ao Estado, a privatização interessava porque o setor público esgotara sua capacidade de investir nas crescentes necessidades de modernização e expansão (LIMA; CARVALHO JR; VELASCO, 1998), principalmente nos setores elétrico, telecomunicação, mineração e infra-estrutura de transporte.

Além disso, a privatização propiciaria a entrada imediata de grande volume de receitas líquidas no setor público e eliminação do custo de endividamento dessas empresas, elementos importantes para revigorar o papel do Estado como indutor do crescimento econômico e para o reequilíbrio das contas públicas. Em relação a esse último aspecto, o IPEA, em documento interno da época da privatização, afirmava que "o processo de privatização tem papel fundamental no aumento da eficiência econômica e na manutenção da estabilidade da relação dívida pública/PIB."

Pelo lado dos compradores privados, a privatização representava oportunidade de potenciais retornos econômicos para o capital investido, sendo exemplos para essa inferência: (i) a pré-existência de um sólido mercado cativo para os produtos gerados pelas empresas privatizadas; (ii) a grande margem de melhoria da eficiência operacional das empresas adquiridas, com impacto importante na redução de custos; e (iii) a economia de imposto de renda e contribuição social sobre o lucro a pagar no futuro, pela aquisição dos créditos tributários e diferimento como despesa dedutível do ágio pago na aquisição.

Nesse contexto, foi elaborado o programa brasileiro de privatização, que 
abrangia: (i) o Programa Nacional de Desestatização - PND, de âmbito federal, iniciado em 1991; (ii) os programas estaduais de privatização, iniciados em 1996; e (iii) o programa de privatização do setor de telecomunicações, também federal, de 1997, conduzido em paralelo com o PND. Conforme Anuatti-Neto et al (2005), considerando apenas os leilões de venda de empresas estatais federais controladas pela união e as participações minoritárias desta em outras empresas, o governo arrecadou US\$ 67,9 bilhões de receitas, mais US\$18,1 bilhões em dívidas transferidas aos compradores, sendo este um dos maiores programas de privatização do mundo.

A atratividade para os compradores pode ser ilustrada pelos elevados ágios pagos sobre os preços mínimos de aquisição. Considerando apenas as empresas de telecomunicações, o ágio total arrecadado foi, segundo Lins (2000), de R\$22,5 bilhões. As regras tributárias vigentes à época permitiam a amortização progressiva desse ágio como despesa dedutível do imposto de renda a pagar, ao longo de um tempo futuro de 10 anos. Conseqüentemente, as empresas pagaram menos imposto de renda após as aquisições realizadas porque seus lucros tributados futuros foram relativamente menores. Este ponto gerou reações negativas sobre o processo de privatização, pois a perda de arrecadação do governo, tudo o mais constante, foi entendida por alguns, conforme Lins (2000), como uma transferência de riqueza da sociedade para os grupos adquirentes. Essa reação pode estar equivocada, pois o sobrevalor desembolsado na aquisição de um ativo com preço mínimo fixado é parte integrante do preço pago pelo investimento e, como tal, deve ser deduzido das receitas tributadas geradas por este, para fins de apuração do lucro tributável.

Ao Banco Nacional de Desenvolvimento Econômico e Social - BNDES coube a tarefa de administrar o programa de privatização brasileiro. Os documentos produzidos por esse banco constituem a principal fonte de informação sobre esse programa. Um balanço por setor mostra, até o ano de 2001, a seguinte distribuição dos leilões de privatização, em termos de valores arrecadados, conforme BNDES (2001): setor elétrico - 31\%; telecomunicações - 31\%; aço - 8\%; mineração - 8\%; óleo e gás - 7\%; petroquímica - 7\%; financeiro $6 \%$; e outros - $2 \%$.

Outro fator importante que impulsionou a demanda para aquisição de ativos estatais era a expectativa que os compradores tinham de promover grandes economias de custo através da melhoria dos processos operacionais e consequiente eliminação de desperdícios, como decorrência do novo modelo de gestão privado e dos investimentos subseqüentes em modernização tecnológica. Este aspecto está ressaltado em quase todos os trabalhos que tratam do processo de privatização brasileiro, sendo exemplos, Rosa e Hoffman (1995), Sampaio, Ramos e Sampaio (2005) e Anuatti-Neto et al (2005).

A comprovação empírica da esperada melhoria da eficiência operacional das empresas estatais após sua privatização não é trivial. As regras contábeis impõem o diferimento dos gastos com investimentos para períodos futuros, de modo a confrontálos progressivamente com a receita realizada, que em geral só é reconhecida no momento em que a empresa vende seus produtos aos consumidores externos. Como a melhoria da eficiência requer altos investimentos em modernização tecnológica, estes se transformam em aumento dos custos operacionais no futuro, por essa razão o confronto do montante absoluto de despesas operacionais, extraído das demonstrações financeiras publicadas, antes e após a privatização, pode não capturar a melhoria da eficiência. Esse problema pode ser contornado, por exemplo, com a 
aplicação de modelos econométricos a variáveis financeiras relativas, extraídas das demonstrações contábeis, e com o uso de metodologias de fronteira de eficiência do tipo Análise Envoltória de Dados - DEA , que focam os processos operacionais em sua dimensão física.

Um exemplo do primeiro caso é a pesquisa de Anuatti-Neto et al (2005), que investigou as diferenças antes e após a privatização de 15 variáveis contábeis relativas, cobrindo aspectos e conceitos relacionados com lucratividade, eficiência operacional, investimento, produção, dividendos, financeiro e impostos. Usando dois métodos de investigação - teste de médias e regressão linear multivariada com dados em painel -, os autores constataram um aumento da lucratividade e da eficiência operacional das empresas da amostra após a privatização.

Entre os estudos que utilizam a metodologia DEA para investigar a eficiência operacional em sua dimensão física, no contexto da privatização, cita-se Sampaio, Ramos e Sampaio (2005), que analisaram o grau de eficiência técnica de um grupo de 71 usinas hidrelétricas públicas e privadas. Os resultados encontrados pelos autores indicaram que o setor público foi mais eficiente do que o privado, destacando-se a Companhia Energética de São Paulo - CESP (empresa pública estadual) e a Light (empresa privada), respectivamente, com o melhor e pior desempenho.

Outra forma de comparar a evolução da eficiência operacional é centrar a análise no comportamento do CPV, total ou em componentes deste. Essa variável expressa o custo da produção, e por isso captura o consumo de todos os recursos, fixos e variáveis, utilizados no processo de geração dos produtos que são vendidos aos consumidores externos à empresa. $O$ foco nesses custos permite ao pesquisador, a um só tempo, observar e comparar o valor monetário dos investimentos em máquinas, mão-de-obra e matéria-prima consumidos na produção de cada período. Logo, se no médio prazo a parcela consumida dos investimentos na modernização tecnológica do parque fabril e dos processos de produção crescesse menos que proporcionalmente ao aumento das receitas estaria havendo aumento de eficiência; o contrário, ineficiência. Nessa linha, Oliveira e Lustosa (2007) examinaram a parcela de custos variáveis presente no CPV da CVRD antes e após a privatização, constatando que houve um aumento da eficiência operacional.

\subsection{A PRIVATIZAÇÃO DA COMPANHIA VALE DO RIO DOCE - CVRD}

Privatizada em 1997, a CVRD é uma das maiores empresas produtoras e exportadoras de minério de ferro do mundo. Foi vendida por US\$ 3,13 bilhões, um preço compatível com o mínimo que fora estabelecido. A propósito do preço mínimo, houve muitas reclamações de que ele teria sido muito baixo porque o banco contratado para determiná-lo (Merryll Linch) deixou de considerar as reservas de minério prospectadas, mas ainda não exploradas, na projeção dos fluxos de caixa futuros da empresa. Este argumento e questionamentos de desvios éticos na condução do processo são motivos de ações ainda vigentes na justiça brasileira pedindo a anulação dessa privatização. 
Depois de privatizada, a empresa cresceu exponencialmente. Em 2010, contabilizava parcerias com a China, negócios na África, exploração de outros negócios (energia elétrica, por exemplo) e outros tipos de metais, aquisição de 16 novas empresas, no Brasil e no exterior, aí incluídas a canadense Inco adquirida em 2006 por US\$ 13 bilhões, o maior negócio já feito por uma empresa da América Latina, e o recolhimento de US\$ 4 bilhões de impostos para o Estado. Com 56 mil empregados e gerando 620 mil empregos indiretos, seu valor de mercado atual é 10 vezes maior do que na época da privatização.

O custo de produção da CVRD equilibra-se entre custos fixos e variáveis e predomina sobre os custos administrativos. A empresa possui grande volume de bens de capital e de mão-de-obra na produção, o que torna seu custo fixo elevado. Mas também consome muitos insumos diretos no processo de extração e beneficiamento dos minérios e demais negócios que explora. Ao constatar que havia uma alta correlação entre os custos de produção da CVRD e sua receita, Oliveira e Lustosa (2007) regrediram o CPV contra o ROL, e com isso segregaram empiricamente o custo variável do custo fixo mostrados de forma agregada no CPV nas demonstrações contábeis publicadas. Um teste de média das séries de custos variáveis antes e após a privatização mostrou que houve redução significativa, em torno de 33\% (o fator de custo variável unitário em relação a ROL passou de 0,8036 a 0,5399), uma evidência de ganhos de eficiência operacional no processo de produção.

\subsection{CUSTOS DE PRODUÇÃO}

Segundo Leone (2000, p. 43), “os chamados fatores de produção e de realização são os materiais, a mão-de-obra e as despesas gerais de fabricação ou de realização. Os fatores de produção representam, também, os recursos disponíveis que serão consumidos pela atividade operacional". A administração da empresa e a contabilidade, tanto financeira quanto gerencial, utilizam técnicas de avaliação e controle diferentes para o consumo de cada um dos três recursos produtivos.

Conforme Garrison e Noreen (2001, p. 28), “materiais diretos são aqueles que se tornam parte integrante do produto acabado e que podem ser física e adequadamente identificados a ele". Para Martins (2003) a mão-de-obra direta é aquela relativa ao pessoal que trabalha diretamente sobre o produto em elaboração, sem necessidade de qualquer apropriação indireta ou rateio. Nesse sentido, os custos de mão-de-obra que não podem ser física e adequadamente identificados às unidades dos produtos, ou que ocasionariam um custo muito elevado para tal adequação, são denominados mão-de-obra indireta, e considerados custos indiretos de fabricação, assim como os materiais indiretos.

O custo indireto de fabricação abrange todos os custos de fabricação, com exceção dos materiais e da mão-de-obra direta. Compreende itens como materiais indiretos, mão-de-obra indireta, manutenção de equipamento de produção, aquecimento e energia, imposto predial, depreciação e seguro das instalações da 
fábrica. Garrison e Noreen (2001, p.28) destacam que "somente os custos relativos à operação da fábrica são incluídos nessa categoria".

Segundo Martins (2003), o custo indireto adicionado à mão-de-obra direta é conhecido como custo de conversão, pois os custos de mão-de-obra direta e os custos indiretos de fabricação ocorrem na transformação dos materiais em produtos acabados. A mão-de-obra direta somada aos materiais diretos é chamada de custo primário. Os custos primários estão relacionados a todos os custos diretos de produção.

Em relação ao comportamento dos custos, Leone (2000, p. 53) explica que "o volume das operações determina os montantes dos custos e das despesas". Nesse âmbito, a Contabilidade de Custos analisa o comportamento dos custos (e das despesas) diante da variação do volume das operações. Para isso, a contabilidade elege determinada unidade de medida do volume, chamada de base de volume (ou base de atividade), e observa como um custo (ou uma despesa) se comporta diante da variabilidade dessa base de volume, classificando-o como fixo, variável ou semivariável.

Os custos variáveis são aqueles que se alteram em função do nível de atividade. O custo variável muda, em seu total, a medida que ocorrem alterações no nível relativo de atividade (HORNGREN, FOSTER e DATAR, 1999). Os custos fixos são custos que não variam com a variabilidade da atividade escolhida, ou seja, o valor total dos custos continua praticamente igual com a variação da base de atividade selecionada (LEONE, 2000).

Em relação aos custos de mão-de-obra, Garrison e Noreen (2001, p. 136) consideram que "o comportamento dos salários muda de um país para outro, dependendo da legislação trabalhista, dos contratos de mão-de-obra e do costume". No Brasil, o custo com a mão-de-obra direta seria melhor classificado como custo fixo, pois a maioria dos empregados é remunerada por um salário fixo e não por hora trabalhada. Além disso, a separação da parcela correspondente ao tempo efetivamente pago na produção, muitas vezes, não é economicamente viável (MARTINS, 2003). Os custos semivariáveis ou custos mistos são os custos que possuem uma parte variável que se comporta como se custo variável fosse e uma parte fixa que se comporta como se custo fixo fosse. (LEONE, 2000).

Com o intuito de analisar a variação dos custos variáveis na CVRD antes e após a privatização, Oliveira e Lustosa (2007) aplicaram o método de análise de regressão a fim de separar os dados dos custos de produção em custos fixos e custos variáveis, já que o CPV é um item de custo que pode ser considerado semivariável.

Os autores utilizaram-se de restrição ao conceito originário de custos primários como forma de alcançar os objetivos propostos. Nesse sentido, os custos variáveis restringiam-se, naquele estudo, aos materiais diretos, o que proporcionou aos pesquisadores obter inferências acerca do desempenho produtivo e operacional da companhia. 


\subsection{MÉTODO DE SIMULAÇÃO NO PROCESSO DECISÓRIO}

A simulação é um instrumento de análise quantitativa utilizado para gerar e analisar alternativas antes de sua implementação. De acordo com Andrade (1989, p.238): "A simulação pode ser usada para experiências com novas situações, sobre as quais se tem pouca ou mesmo nenhuma informação, com o intuito de preparar a administração para o que possa acontecer.

Assim, a simulação pode representar um fator positivo na tomada de decisões, incluindo sua aplicação nos processos de privatização, uma vez que permite a realização de inferências, por meio de experimentos, sobre o comportamento das variáveis envolvidas na decisão. Proporciona à direção a possibilidade de examinar e avaliar diversos planos muito antes de acatar projetos importantes. Uma vez determinado o plano mais conveniente, aquele que contém o máximo de vantagens e o mínimo de desvantagens, pode-se por em prática na situação real (ESCUDERO, 1973).

Existem dois tipos de modelos de simulação: o determinístico e o probabilístico. Nos modelos determinísticos, segundo Reis e Martins (2001, p. 58), "pressupõe-se que os dados são obtidos com certeza", ou seja, não incorpora as probabilidades de que o valor escolhido para a simulação sofra alterações futuras. Já o segundo modelo incorpora o comportamento probabilístico no relacionamento interno do sistema, na tentativa de capturar a natureza probabilística envolvida nas variáveis que cercam o sistema, por meio da utilização da técnica estatística e do uso de computadores (NASCIMENTO; ZUCCHI, 1997).

Os modelos de simulação probabilísticos tiveram sua origem no método de Monte Carlo e têm como foco simulações de fenômenos aleatórios, introduzindo a análise de riscos, incorporando as variáveis ambientais e, conseqüentemente, os elementos de incerteza inerentes (NASCIMENTO; ZUCCHI, 1997).

A Simulação de Monte Carlo tem este nome devido à famosa roleta de Monte Carlo, no Principado de Mônaco. Seu nome, bem como o desenvolvimento sistemático do método, data de 1944, quando da Segunda Guerra Mundial, período em que foi ferramenta de pesquisa para o desenvolvimento da bomba atômica.

Hammersley e Handscomb (1964, p. 3), definem o Método de Monte Carlo como sendo "a parte da matemática experimental que está preocupada em experiências com números aleatórios". O Método de Monte Carlo é uma expressão muito geral, onde as formas de investigação estão baseadas no uso de números fortuitos e estatística de probabilidade. 


\section{PROCEDIMENTOS METODOLÓGICOS}

\subsection{SIMULAÇÃO}

De acordo com Andrade (1989), as fases para realização de uma simulação compreendem: a Formulação do Problema; a Coleta de Dados; a Identificação das Variáveis aleatórias que serão simuladas e suas respectivas distribuiçõos de probabilidades; A Formulação do Modelo, com a modelagem das relações entre as variáveis do problema; a Avaliação do Modelo e a Realização dos Experimentos de Simulação.

Neste trabalho, a formulação do problema consiste na utilização do Método de Simulação de Monte Carlo para simular os custos de produção da CVRD no período pós-privatização. 0 objetivo é empregar a simulação em duas situações: aplicação do método sem considerar qualquer hipótese adicional relativa à privatização; e aplicação do método considerando a hipótese de crescimento anual da ROL e decrescimento inicial dos custos dos produtos vendidos. A primeira aplicação, ao desconsiderar qualquer hipótese adicional, verifica como estariam os custos de produção da CVRD se a mesma não tivesse sido privatizada.

Os dados foram coletados das demonstrações financeiras publicadas pela Companhia no período de 1990 a 1996 (primeira amostra). O ano de 1997 foi o ano da privatização e, com isso, não foi incluído na amostra.

As variáveis aleatórias consideradas são CPV e ROL Na primeira aplicação são utilizados os dados de CPV da primeira amostra para simular o CPV no período pós privatização, compreendendo os anos de 1998 a 2004. Na segunda aplicação são utilizados os dados de ROL da primeira amostra para simular o CPV no período de 1998 a 2004. Neste caso, é utilizada a inferência de que o CPV é uma função linear do ROL e os parâmetros da relação foram obtidos por meio de uma regressão linear no trabalho de Oliveira e Lustosa (2007). Assim, a modelagem do problema compreende somente a definição do relacionamento entre as variáveis de CPV e ROL, conforme a equação a seguir que demonstra o comportamento do CPV em relação à ROL no período pós-privatização:

$$
\text { CPV }=-45530+0,5399 \times R O L
$$

Dessa forma, o CPV é obtido a partir do ROL simulado para cada um dos 28 trimestres do período de 1998 a 2004. Também é considerado um crescimento anual de $15 \%$ sobre os valores de ROL simulados, conforme dados históricos de incremento de vendas da Companhia no período pós-privatização. Neste caso, é importante observar que foram escolhidos estes parâmetros porque já foram objeto de testes estatísticos em trabalho anterior. Uma boa definição do relacionamento entre as variáveis do problema, bem como, a acurácia na estimativa de outros parâmetros do modelo contribuirão para aferir, sem vieses, sobre o desempenho do Método de Monte Carlo nesta situação específica, o que é o objetivo deste trabalho. 
Para realização do experimento, o primeiro passo é a identificação das distribuições de probabilidades das variáveis aleatórias definidas no modelo. De acordo com Andrade (1989), o Método de Monte Carlo baseia-se num conceito estatístico simples. A função cumulativa de probabilidades da variável aleatória $X$ tem uma distribuição uniforme sobre o intervalo fechado $(0,1)$. Então, dada a função de distribuição de probabilidades da variável em simulação e a sua respectiva função cumulativa de probabilidades, é tomado um número gerado aleatoriamente no intervalo $(0,1)$. Usando a função cumulativa, é determinado o valor da variável $X$ que corresponde ao número aleatório gerado.

Ressalta-se que as populações analisadas devem ter certos parâmetros, como média e desvio padrão, e podem apresentar vários comportamentos como Normal, Exponencial e Uniforme. As amostras obtidas devem ser aleatórias. Para isso, é preciso obter uma sequiência de números aleatórios. Computacionalmente, esta seqüência é facilmente obtida, utilizando um gerador de números aleatórios. Os números gerados são pseudo-aleatórios, mas, geralmente, são suficientes para obter aproximações razoáveis de números aleatórios inteiros e podem ser utilizados para obter amostras aleatórias de alguma população de interesse.

São utilizadas séries de tentativas aleatórias e a precisão do resultado final depende, em geral, do número de tentativas. O equilíbrio entre a precisão do resultado e o tempo de computação é uma característica útil dos métodos de Monte Carlo (ESCUDERO, 1973). Segundo Lustosa, Ponte e Dominas (2004), para a efetivação da simulação, deve-se encontrar cem números aleatórios, no mínimo, para cada um dos resultados almejados, pois quanto maior for a amostra, melhores os resultados obtidos.

Neste trabalho, com base em análises dos dados históricos, constatou-se que o comportamento das variáveis aleatórias CPV e ROL se aproxima da distribuição normal. Para esta distribuição de probabilidades, o Excel $^{1}$ possui uma função estatística programada denominada INV.NORM, a qual retorna o inverso da distribuição cumulativa normal para a média e o desvio padrão especificados (LUSTOSA; PONTE; DOMINAS, 2004). Também os números aleatórios são gerados no Excel pela função ALEATORIO().

Assim, para cada amostra analisada é necessário calcular a média e o desvio padrão e, usando a fórmula a seguir, são gerados os números aleatórios e respectivos valores das variáveis aleatórias $\mathrm{x}$ :

$$
\text { =inv.norm(aleatório();célula da média;célula do desvio padrão) }
$$

Na primeira aplicação, os dados de CPV de 28 trimestres antes da privatização foram simulados aleatoriamente para mais 28 trimestres, ou seja, sete anos. Para cada trimestre simulado, foram calculados, usando a fórmula (2) acima, 100 números aleatórios. A média desses valores foi considerada o valor esperado e adicionada à amostra de CPV, alterando a quantidade de observações, a média aritmética bem como o desvio padrão da distribuição, para a obtenção do próximo trimestre e assim

1 Software marca registrada da Microsoft ${ }^{\circledR}$ Corporation (Planilhas eletrônicas). 
por diante. Dessa forma, para essa aplicação, foram elaborados 2.800 números aleatórios.

Na segunda aplicação os dados de ROL de 28 trimestres pré-privatização foram simulados para mais 28 trimestres pós-privatização. Da mesma forma que a outra aplicação, foram elaborados 100 números aleatórios para cada trimestre simulado de ROL e a média foi novamente considerada o valor esperado. Após a obtenção dos 28 trimestres, foi adicionado aos valores simulados, $15 \%$ de crescimento esperado a cada período. Dessa maneira, para a realização da segunda aplicação, foram elaborados mais 2.800 números aleatórios, totalizando 5.600 números aleatórios pelo Método de Monte Carlo.

Para introduzir a hipótese adicional de redução de custos, o CPV de cada trimestre foi calculado a partir do ROL simulado para cada trimestre, com o incremento de $15 \%$, usando os parâmetros da regressão linear mostrados na equação (1).

Após a simulação em cada uma das situações, as amostras reais e simuladas foram analisadas quantitativamente por meio da estatística descritiva, comparando as situações antes e após a privatização. Foram efetuados testes de médias para cada situação, com o intuito de verificar a existência de diferenças significativas entre as amostras reais e simuladas.

\section{RESULTADOS E ANÁLISES}

\subsection{PRIMEIRA APLICAÇÃO: SIMULAÇÃO DO CPV SEM CONSIDERAR QUALQUER HIPÓTESE ADICIONAL}

Foram utilizados como base os valores dos CPV no período antes da privatização (1990 a 1996) e, a partir da aplicação do Método de Monte Carlo, foram encontrados 28 valores simulados que se referem ao período pós-privatização. Os valores simulados da presente situação não consideram o fator desestatização. Então os resultados encontrados simulam a provável situação dos custos de produção da CVRD se a mesma ainda fosse estatal.

Dessa forma, era provável que os custos simulados se diferenciassem dos custos reais, tendo em vista a desconsideração de um efeito tão relevante. Essa situação é ilustrada nos gráficos 1 e 2 . Os dados que os geraram referem-se ao CPV real do período pós-privatização e ao CPV simulado do mesmo período, respectivamente. 


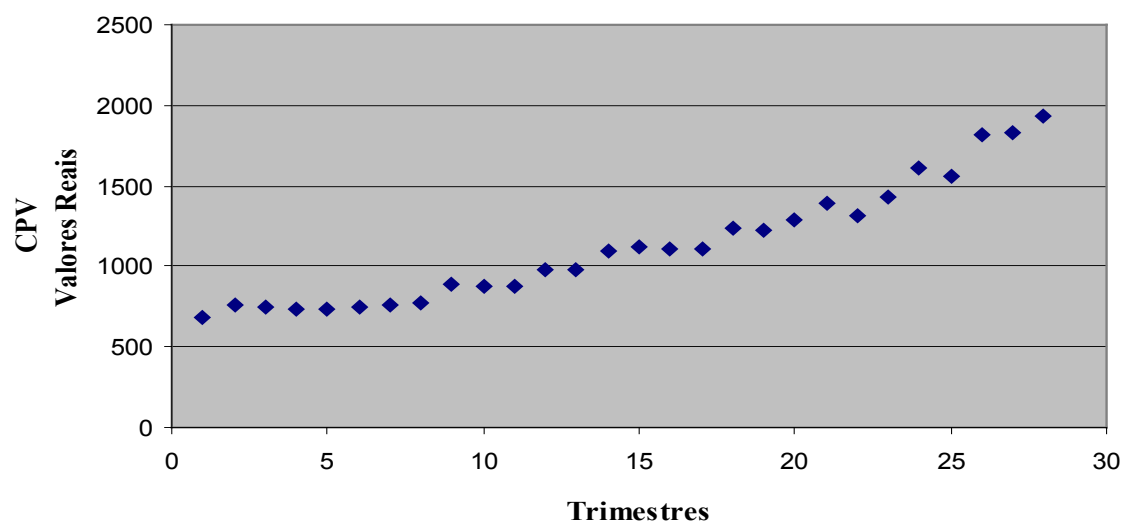

Gráfico 1 - CPV real X Tempo (R\$ Milhões)

Período pós-privatização (1998 a 2004)

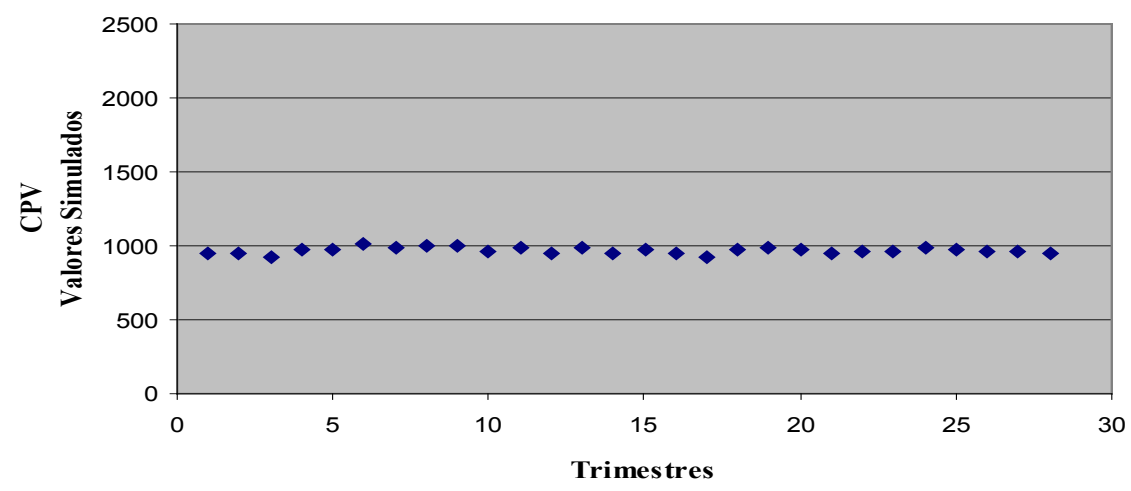

Gráfico 2 - CPV simulado X Tempo (R\$ Milhões)

Período pós-privatização (1998 a 2004)

Os gráficos foram elaborados com a mesma escala. Isso resulta em maior facilidade de comparação entre as amostras nessas circunstâncias, evitando a interpretação errônea (TOLEDO; OVALLE, 1992). As estatísticas descritivas das amostras são apresentadas na Tabela 1, a seguir:

Tabela 1 - Estatísticas Descritivas

CPV reais e simulados sem considerar hipótese adicional

\begin{tabular}{c|c|c}
\hline & DADOS REAIS & DADOS SIMULADOS \\
\hline Média & $1,129 \mathrm{E}+06$ & $9,668 \mathrm{E}+05$ \\
\hline Mediana & $1,105 \mathrm{E}+06$ & $9,663 \mathrm{E}+05$ \\
\hline Desvio Padrão & $3,720 \mathrm{E}+05$ & $2,279 \mathrm{E}+04$ \\
\hline Variância da amostra & $1,384 \mathrm{E}+11$ & $5,194 \mathrm{E}+08$ \\
\hline Assimetria & 0,72 & 0,18 \\
\hline Amplitude & $1,255 \mathrm{E}+06$ & $9,381 \mathrm{E}+04$ \\
\hline Mínimo & $6,828 \mathrm{E}+05$ & $9,235 \mathrm{E}+05$ \\
\hline Máximo & $1,938 \mathrm{E}+06$ & $1,017 \mathrm{E}+06$ \\
\hline Coeficiente de Variação & 0,33 & 0,02
\end{tabular}

A média dos valores reais é, aproximadamente, 17\% maior que a média dos valores simulados. Essa medida, tomada isoladamente, pode não ser relevante, tendo em vista que ela não fornece informações sobre outros aspectos da distribuição, como por exemplo a variabilidade da série. 
Uma medida bastante utilizada na análise da variabilidade de uma variável é o desvio padrão. Este será tão maior quanto mais dispersos forem os valores observados (BARBETTA, 2003). Nessa análise, a amostra de valores reais apresenta um desvio muito maior em relação à outra amostra, significando maior dispersão de seus valores, o que pode ser visualmente observado no gráfico 1.

Em relação à mediana, a amostra de valores reais é aproximadamente $14 \%$ maior que a de valores simulados. A mediana é o valor que divide uma série ordenada de tal forma que pelo menos a metade seja igual ou maior do que ela. É uma medida muito utilizada na análise de dados estatísticos, principalmente ao não se atribuir importância aos valores extremos da variável (TOLEDO; OVALLE, 1992).

O intervalo ou amplitude se refere à diferença entre o ponto máximo e o mínimo. Na observação dessas estatísticas, percebe-se a grande distância entre os pontos extremos dos valores reais com os simulados, bem como a grande diferença em suas amplitudes.

Por último, o coeficiente de variação é decorrente da divisão do desvio padrão pela média. Ele é utilizado para tornar comparáveis as distribuições (BRAULE, 2001). Diante dos resultados, verifica-se que a variabilidade da amostra de valores simulados é de $2 \%$, enquanto a variabilidade da amostra com dados reais é de $33 \%$, sugerindo maior homogeneidade nos dados simulados.

Analisando o conjunto dessas medidas, têm-se respostas preliminares para inferir que a amostra simulada para o período pós-privatização - sem considerar hipótese adicional - se distancia substancialmente dos dados reais, revelando que a privatização é um fator importante que não pode ser desconsiderado nas projeções. Inferências estatísticas, levando em consideração distribuição de probabilidades e testes de hipóteses, serão fundamentais para a obtenção de respostas mais conclusivas acerca das considerações apresentadas.

\subsubsection{TESTE DE MÉDIAS DA PRIMEIRA APLICAÇÃO}

\subsubsection{TESTE $F$ PARA DIFERENÇAS ENTRE DUAS VARIÂNCIAS}

O teste de médias (com elaboração de hipóteses) oferece uma abordagem confirmatória para a análise de dados. Para tanto, é necessário testar se as duas amostras independentes possuem a mesma variabilidade. Uma importante razão para testar a diferença entre as variâncias das duas amostras é a necessidade de determinar se o teste $t$ a ser empregado é o de variâncias agrupadas ou separadas (LEVINE et al, 2005).

O teste para a diferença entre as variâncias das duas amostras é baseado na razão entre as variâncias das amostras, e segue uma distribuição conhecida como distribuição $F$. Nessa primeira etapa, define-se como hipótese nula a existência de igualdade entre as variâncias. A hipótese nula é rejeitada se a estatística do teste $F$ calculada for maior do que o valor crítico da cauda superior ou se posicionar abaixo do valor crítico da cauda inferior da distribuição (teste bicaudal). 
Os resultados do primeiro teste estatístico sugerem a rejeição, com bastante folga, da hipótese nula de igualdade de variâncias, o que indica a utilização do teste $t$ para variâncias distintas quando da comparação das médias amostrais [estatística $F=266,41, F$ crítico superior $=2,78, F$ crítico inferior $=0,36$, significativo a $99 \%$ de nível de confiança].

\subsubsection{TESTE DE MÉDIAS PARA VARIÂNCIAS NÃO AGRUPADAS}

Conforme indicado pelo teste $F$ para diferenças entre variâncias, procedeu-se à análise das diferenças entre as médias aritméticas das séries amostrais com a utilização do teste $t$ para variâncias separadas. A hipótese nula a ser testada, nesse caso, refere-se à igualdade entre as médias aritméticas das duas distribuições amostrais (CPV real e simulado). A tabela 2 a seguir resume os resultados do teste de médias.

Tabela 2 - Teste t presumindo variâncias diferentes

CPV real X CPV simulado (sem hipótese adicional)

\begin{tabular}{c|c|c}
\hline & CPV real & CPV simulado \\
\hline Média & $1,129 \mathrm{E}+06$ & $9,668 \mathrm{E}+05$ \\
\hline Variância & $1,384 \mathrm{E}+11$ & $5,194 \mathrm{E}+08$ \\
\hline Graus de liberdade & 27 & \\
\hline Estatística $t$ & $2,3085^{*}$ & \\
\hline $\mathrm{P}(\mathrm{T} \leq t)$ bicaudal & 0,0289 & \\
\hline$t$ crítico bicaudal & 2,0518 & \\
\hline
\end{tabular}

(*) Significativo a 95\% de nível de confiança.

A utilização do $t$ crítico bicaudal refere-se à forma de elaboração das hipóteses² do teste. De acordo com a hipótese alternativa, a média do CPV real é diferente da média do CPV simulado, o que justifica a utilização do teste $t$ com duas regiões críticas ou de rejeição. Os resultados do teste sugerem a rejeição da hipótese nula de igualdade entre as médias aritméticas, com p-value de 2,89\% contra um nível de significância de $5 \%$. Os resultados obtidos corroboram as expectativas iniciais, uma vez que foi desconsiderado um fator relevante quando da simulação dos valores para o período de 1998 a 2004.

\subsection{SEGUNDA APLICAÇÃO: SIMULAÇÃO DO CPV CONSIDERANDO O CONTÍNUO CRESCIMENTO DO ROL E O DECRESCIMENTO INICIAL DO CPV}

Nessa aplicação, utilizou-se como base os 28 valores da ROL no período antes da privatização. Com o auxílio da Simulação de Monte Carlo e a consideração da hipótese de $15 \%$ de crescimento anual da variável a partir da média simples da variação percentual da receita nos anos subseqüentes, foram encontrados 28 valores simulados de ROL para o período pós-privatização. Como o objetivo deste trabalho não é a comparação da ROL, e sim do CPV, não será apresentada a estatística descritiva dos valores de ROL.

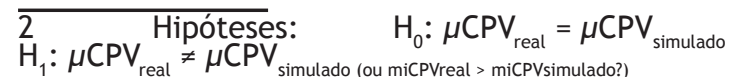


Para atribuir validade empírica às afirmações, efetuou-se o teste de médias (teste $t$ para variâncias equivalentes após a aplicação do teste $F$ ) com o intuito de verificar estatisticamente o pressuposto inicial de existência de igualdade de média entre as duas distribuições de ROL (real e simulada). Os resultados obtidos corroboram com a hipótese inicial, o que indica uma similitude entre os valores reais e simulados de ROL no período posterior à privatização [estatística $t$ de -0,997 contra um $t$ crítico bi-caudal de 2,004].

Partindo dos dados simulados da ROL, foram calculados, por intermédio da equação encontrada no artigo de Oliveira e Lustosa (2007), o CPV, levando-se em consideração a hipótese de relacionamento linear entre as variáveis identificadas. Os coeficientes da equação regredida utilizados foram os calculados para o período pós-privatização e a variável ROL se refere aos valores simulados por intermédio do método. É importante ressaltar que as hipóteses adicionais tiveram como base dados reais pós-privatização, tendo em vista que o objetivo desse trabalho é essencialmente testar a aplicabilidade do método e não a previsão de crescimento da variável. Um equívoco na previsão poderia resultar em uma conclusão errônea em relação à adequação do método.

Os valores simulados nessa segunda aplicação estão mais próximos dos valores reais, o que pode ser constatado na visualização dos gráficos 5 e 6:

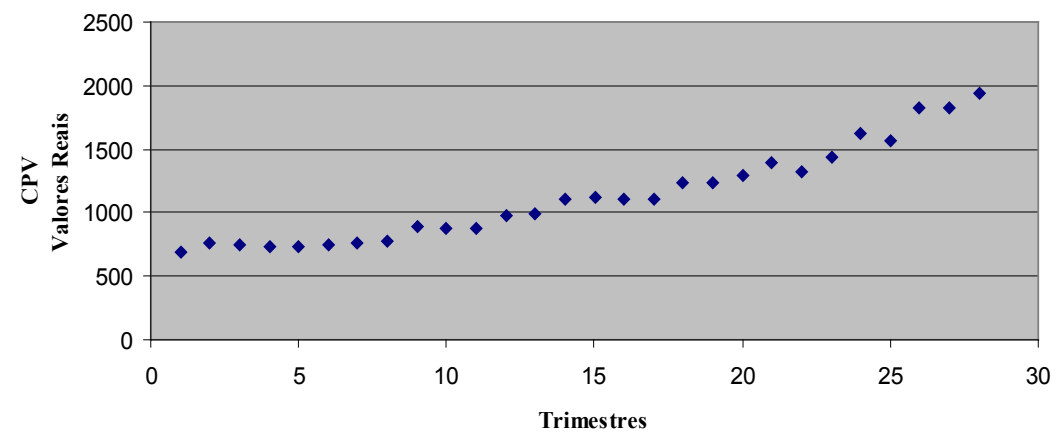

Gráfico 5 - CPV real X Tempo (R\$ Milhões)

Período pós-privatização (1998 a 2004)

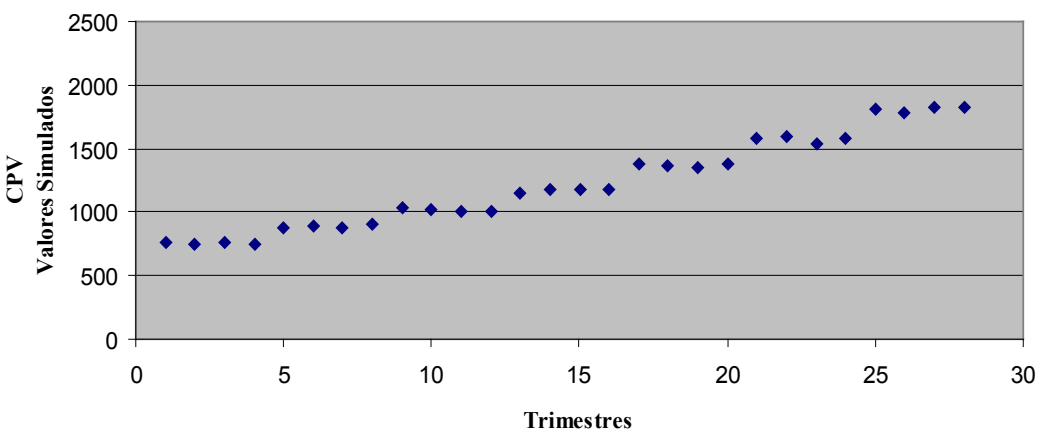

Gráfico 6 - CPV simulado X Tempo (R\$ Milhões)

Período pós-privatização (1998 a 2004)

As escalas de todos os gráficos de CPV são idênticas, tendo como objetivo 
comparar as duas aplicações do Método de Monte Carlo. Na tabela 3 são apresentadas as estatísticas descritivas dos dados reais e simulados nessa aplicação.

Tabela 3 - Estatísticas Descritivas

CPV reais e simulados considerando hipóteses adicionais

\begin{tabular}{c|c|c}
\hline & DADOS REAIS & DADOS SIMULADOS \\
\hline Média & $1,129 \mathrm{E}+06$ & $1,225 \mathrm{E}+06$ \\
\hline Mediana & $1,105 \mathrm{E}+06$ & $1,177 \mathrm{E}+06$ \\
\hline Desvio Padrão & $3,720 \mathrm{E}+05$ & $3,589 \mathrm{E}+05$ \\
\hline Variância da amostra & $1,384 \mathrm{E}+11$ & $1,288 \mathrm{E}+11$ \\
\hline Amplitude & $1,255 \mathrm{E}+06$ & $1,083 \mathrm{E}+06$ \\
\hline Mínimo & $6,828 \mathrm{E}+05$ & $7,476 \mathrm{E}+05$ \\
\hline Máximo & $1,938 \mathrm{E}+06$ & $1,831 \mathrm{E}+06$ \\
\hline Coeficiente de Variação & 0,33 & 0,29 \\
\hline
\end{tabular}

A primeira medida estatística da tabela 3, a média aritmética, já evidencia uma aproximação do CPV simulado ao CPV real, pelo menos no que se refere à posição central. A média da amostra simulada é apenas $8 \%$ maior que a outra. Da mesma forma, as medianas estão mais próximas uma da outra do que na situação anterior.

Há também forte aproximação dos desvios padrão e das variâncias das amostras, significando que as distribuições estão dispersas de forma parecida (em torno de suas respectivas médias). Dessa maneira, é possível entender que o coeficiente de variação da amostra simulada deve ter um valor próximo ao valor da amostra real, tendo em vista a proximidade das médias e dos desvios padrão.

Ao contrário da aplicação que não considera hipótese adicional, as medidas de amplitude estão muito próximas uma da outra na segunda aplicação. Verifica-se também a adequada correspondência dos valores mínimos e valores máximos das amostras. Assim, pode-se considerar que a amostra simulada para o período pósprivatização, levando-se em consideração a tendência de crescimento percentual da ROL e o decrescimento dos custos variáveis, com consequente redução inicial do CPV, foi apropriada, tendo em vista a consideração dos efeitos da privatização, fator que aqui se mostra relevante.

\subsubsection{TESTE DE MÉDIAS DA SEGUNDA APLICAÇÃO}

\subsubsection{TESTE $F$ PARA DIFERENÇAS ENTRE DUAS VARIÂNCIAS}

Conforme discutido anteriormente, o teste $F$ foi utilizado como forma de identificar diferenças ou similitudes entre as variâncias das duas amostras. Os resultados obtidos serviriam de base para decisão em adotar o teste $t$ para diferenças de médias aritméticas com variâncias agrupadas ou separadas.

As análises dos resultados obtidos sugerem a não rejeição da hipótese nula de igualdade entre as variâncias das duas amostras [estatística $F=1,074, F$ crítico 
superior $=2,777, F$ crítico inferior $=0,360, p$-value de 0,853 , significativo a $99 \%$ de nível de confiança]. Dessa forma, o teste $t$ a ser empregado para verificar a diferença entre médias é o de variâncias agrupadas, discutido na seção a seguir.

\subsubsection{TESTE DE MÉDIAS PARA VARIÂNCIAS AGRUPADAs}

A tabela 5 a seguir resume os resultados do teste de médias sobre as amostras de CPV real e simulado, considerando o fator privatização.

Tabela 5 - Teste t presumindo variâncias agrupadas

CPV real X CPV simulado considerando hipóteses adicionais

\begin{tabular}{c|c|c}
\hline & CPV real & CPV simulado \\
\hline Média & $1,129 \mathrm{E}+06$ & $1,225 \mathrm{E}+06$ \\
\hline Variância & $1,384 \mathrm{E}+11$ & $1,288 \mathrm{E}+11$ \\
\hline Observações & 28 & 28 \\
\hline Variância agrupada & $1,336 \mathrm{E}+11$ & \\
\hline Graus de liberdade & 54 & \\
\hline Estatística $t$ & $-0,9808^{* *}$ & \\
\hline P(T $\leq$ ) bicaudal & 0,3310 & \\
\hline$t$ crítico bicaudal & 2,6700 & \\
\hline
\end{tabular}

$\left(^{* *}\right)$ Significativo a $99 \%$ de nível de confiança.

A estatística do teste requer que as duas variâncias das amostras sejam agrupadas ou combinadas no sentido de obter o melhor estimador da variância comum a ambas as amostras, sob a premissa de que as duas variâncias sejam iguais (LEVINE et al, 2005). Esta hipótese pôde ser comprovada por intermédio de teste $F$ discutido na seção anterior.

Assim, os resultados do teste, nessa segunda aplicação, sugerem a não rejeição da hipótese nula de igualdade das médias aritméticas das duas distribuições amostrais de CPV [estatística $t$ de $-0,98$, com $t$ crítico bicaudal de 2,67, ao nível de significância de 1\%]. Os resultados também dão suporte às suposições iniciais de igualdade entre valores reais e simulados quando considerado o fator desestatização, contribuindo assim para o alcance do objetivo proposto e, ainda, solução da questão problema dessa pesquisa.

Verifica-se então uma adequação do Método de Simulação de Monte Carlo na previsão dos custos de produção da CRVD, aliado às ferramentas de análise de regressão e os resultados obtidos por Oliveira e Lustosa (2005), revelando, para esse estudo, a validade das premissas adotadas pelos autores.

\subsection{TESTES DE ROBUSTEZ}

Foram realizados testes Jarque-Bera - JB para verificar a premissa de 
normalidade das amostras, uma vez que os testes de médias e variâncias pressupõem normalidade das séries em análise. O teste estatístico JB segue uma distribuição Quiquadrado $\left(X^{2}\right)$, sob a hipótese nula de que as distribuições das séries são simétricas e mesocúrticas, ou seja, normais (BROOKS, 2002).

Os resultados encontrados sugerem, em todas as amostras, a não rejeição da hipótese nula de normalidade das séries, ao nível de significância de 1\% [CPV real - Jarque-Bera de 2,558 e p-value de 0,278 em ambas as amostras; CPV simulado Jarque-Bera de 0,243 e $p$-value de 0,886, sem considerar hipótese adicional e, JarqueBera de 1,999 e p-value de 0,368, considerando os efeitos da desestatização].

\section{CONSIDERAÇÕES FINAIS}

As decisões no mundo dos negócios, de comprar e vender ativos, ou de transformá-los em outros no processo de manufatura, ocorrem em um ambiente de incertezas. A continuidade da empresa no mercado competitivo depende do grau de acerto das decisões do seu gestor, que pode ser medido pela maior ou menor proximidade do cenário antecipado no momento corrente com o que efetivamente ocorrerá no futuro.

Assim, a antecipação de cenários sobre o futuro incerto é um imperativo no ambiente empresarial e para cumprir essa função os gestores adotam diversos tipos de métodos, que variam desde a confiança na sua própria experiência e intuição, até o uso sistematizado de técnicas quantitativas.

Uma das técnicas quantitativas que pode ser usada para essa finalidade é a Simulação de Monte Carlo. Essa técnica se apóia numa premissa de aleatoriedade, ou imprevisibilidade dos fenômenos, segundo a qual não é possível antecipar com certeza o valor de uma variável, mas apenas anunciar a probabilidade de ocorrência do seu valor esperado, o que depende da distribuição de freqüência da variável.

Nesse trabalho, testou-se a aplicabilidade do método de simulação de Monte Carlo para prever como seria o comportamento da variável CPV, da CVRD, após a privatização dessa companhia que ocorreu em 1997. Os testes foram realizados em duas situações. A primeira, utilizou uma série histórica da variável CPV antes da privatização, de 1990 a 2006 e, em um experimento de 100 simulações trimestrais, assumindo uma distribuição normal dessa variável, projetou-se como seria o comportamento do CPV da companhia de 1998 a 2004 (2800 simulações no total). Os resultados do CPV obtidos com essa simulação foram estatisticamente comparados, por meio de um teste paramétrico de médias, com os dados reais da mesma variável. Esperava-se que os parâmetros das duas amostras seriam significativamente diferentes, já que a privatização da CVRD produziria uma mudança no comportamento dos custos de produção da empresa. O resultado confirmou a expectativa de que de fato existiu uma diferença significativa entre da média e variabilidade da amostra simulada em relação aos valores desses mesmos parâmetros para a amostra real, o que valida o teste da aplicabilidade da simulação de Monte Carlo nessas situações. 
A segunda simulação, também de 100 repetições por trimestre num total de 2800 repetições, se apoiou na pesquisa de Oliveira e Lustosa (2007), que encontraram evidências empíricas de que, no período 1998-2004, houve uma redução média de $33 \%$ nos custos variáveis de produção da CVRD quando comparado com igual período anterior à sua privatização. Os estimadores da regressão linear do CPV contra o ROL obtidos pelos autores foram utilizados para simular o CPV, com a condição adicional, também baseada nessa mesma pesquisa, de que a receita pós-privatização crescia a uma taxa média de $15 \%$ ao trimestre. Como essa segunda simulação considerava a realidade existente após a privatização, não se poderia esperar diferenças significativas entre os dados do CPV reais e simulados. 0 teste de média das duas séries de amostra de CPV, real e simulada, mostrou que suas médias e variabilidade eram estatisticamente iguais, confirmando, mais uma vez, a aplicabilidade do método de simulação de Monte Carlo para projetar cenários de custos de produção.

As duas simulações do CPV apresentadas neste trabalho se basearam em dados ex post. Na primeira, assumiu-se que o comportamento médio do CPV verificado no passado iria se repetir no futuro. Na segunda, os estimadores do CPV obtidos empiricamente com base no comportamento real do CPV e do ROL da companhia ao longo do período amostral foram utilizados para realizar a simulação. 0 uso de informações já constatadas na realidade possibilitou aferir sobre o desempenho do método, permitindo concluir que a eficácia das técnicas de simulação é diretamente proporcional ao nível de acurácia com que se consegue reproduzir, no presente, o comportamento esperado das variáveis que serão objeto de simulação. Assim, em um ambiente de decisão, onde os parâmetros têm que ser definidos à priori, os esforços recaem na experiência da administração, nos referenciais de mercado, na análise de cenários ou na utilização de métodos quantitativos para auxiliar o processo de estimativas sobre o comportamento das variáveis.

A partir dessas constatações, pode-se inferir que o objetivo proposto foi alcançado. A Simulação de Monte Carlo mostrou-se um método apropriado para prever os custos de produção da CVRD, se adequando aos impactos do fator modificativo desestatização. Sendo assim, o método apresenta-se como possível ferramenta no auxílio à visualização de diversas situações, inclusive contrastantes, nos processos de decisão.

\section{REFERÊNCIAS}

ANDRADE, E. L. Introdução à Pesquisa Operacional - Métodos e Modelos para a Análise de Decisão. Rio de Janeiro: LTC - Livros Técnicos e Científicos Ed., 1989.

ANUATTI-NETO, F.; BAROSSI-FILHO, M; CARVALHO, A. G.; MACEDO, R. Os Efeitos da Privatização sobre o Desempenho Econômico e Financeiro das Empresas Privatizadas. Revista Brasileira de Economia. Rio de janeiro: 59(2), abr/jun 2005, p. 151-175.

BARBETTA, P. A. Estatística aplicada às Ciências Sociais. 5 ed. Florianópolis: Ed. Da 
UFSC, 2003.

BNDES. Privatização. 2001. Disponível em www.bndes.gov.br

BRAULE, R. Estatística aplicada com Excel. São Paulo: Campus, 2001.

BROOKS, C. Introductory Econometrics for Finance. Cambridge: Cambridge University Press, 2002.

CHAN, B. L.; SANTOS, A.; SILVA, F. L. Uma contribuição ao estudo dos impactos da privatização à luz da Demonstração do Valor Adicionado. In: $3^{\circ}$ CONGRESSO USP DE CONTROLADORIA E CONTABILIDADE, São Paulo, 2003. Disponível em: http://www. eac.fea.usp.br/congressousp/congresso3. Acesso em: 10 set. 2006.

COLODETI FILHO, E.; GOMES, C. E. A.; TEIXEIRA, A. J. C. Uma reflexão sobre a segregação dos custos com o uso da análise de regressão linear: o caso da Espírito Santos Borrachas. In: $3^{\circ}$ CONGRESSO USP DE CONTROLADORIA E CONTABILIDADE, São Paulo, 2003. Disponível em: http://www.eac.fea.usp.br/congressousp/ congresso3. Acesso em: 10 set. 2006.

ESCUDERO, L. F. La simulación en la empresa. Barraincúa: Deusto, 1973.

GARRISON, R.H.; NOREEN, E.W. Contabilidade Gerencial. 9a Ed. Rio de Janeiro: LTC, - Livros Técnicos e Científicos Ed., ., 2001. Tradução.

HAMMERSLEY, J.M e HANDSCOMB, D. C. Monte Carlo methods. London: Methuen, 1964.

HORNGREN, C., FOSTER, G. e DATAR, S. M. Cost Accounting: A Managerial Enphasis. Prentice Hall, 10ª edição, 1999.

IPEA - Instituto de Pesquisas Econômicas Aplicadas. IPEA Publicações. Ver em http://www.ipea.gov.br/pub/boletim/bfpempre.html. Consulta realizada em 10/02/2011.

LEONE, G. S. G. Custos, Planejamento, Implantação e Controle. São Paulo: Ed. Atlas, $3^{\mathrm{a}}$.edição, 2000.

LEVINE, D. M.; STEPHAN, D.; KREHBIEL, T. C.; BERENSON, M. L. Estatística: Teoria e aplicações - Usando Microsoft Excel em português. Rio de Janeiro: LTC, 2005.

LIMA, E. T.; CARVALHO JR, M. C.; VELASCO, L. O. M. Removendo Obstáculos às Exportações Brasileiras. Publicação interna do BNDES, 06/1998. Ver em www.bndes. gov.br.

LINS, B. E. Privatização das Telecomunicações Brasileiras: algumas lições. Cadernos Aslegis, 4(10), 2000, p. 9-25.

LUSTOSA, P. R. B.; PONTE, V. M. R.; DOMINAS, W. R. Simulação. In: CORRAR, L. J; THEÓPHILO, C. R. (Coord.). Pesquisa operacional para decisão em contabilidade e administração: contabilometria. São Paulo: Atlas, 2004. P. 242-284. 
MARTINS, E. Contabilidade de Custos. 10 Ed. São Paulo: Atlas; 2003.

NASCIMENTO, A. M.; ZUCCHI, A. L. Modelos de simulação. São Paulo, Universidade de São Paulo, 1997. 40 p. Monografia - Faculdade de Economia, Administração e Contabilidade, Universidade de São Paulo, São Paulo, 1997.

OLIVEIRA, F. L. J.; LIMA, A. V. Um estudo do desempenho de empresas brasileiras privatizadas no período de 1991 a 1997 sob a ótica do valor adicionado. In: $2^{\circ}$ SEMINÁRIO USP DE CONTABILIDADE, São Paulo, 2002. Disponível em: http://www. eac.fea.usp.br/congressousp/seminario2. Acesso em: 10 set. 2006.

OLIVEIRA, P. H. D.; LUSTOSA, P. R. B. Custos primários como parâmetro de eficiência produtiva: uma análise empírica da Companhia Vale do Rio Doce antes e após a privatização. Revista Universo Contábil, Blumenau: v. 3, n. 3, p. 54-70, set./ dez. 2007.

REIS, S. G.; MARTINS, E. Planejamento do balanço bancário:desenvolvimento de um modelo matemático de otimização do retorno econômico ajustado ao risco. Revista Contabilidade \& Finanças, São Paulo, v. 15, n. 26, p.: 58-80, Maio/Agosto de 2001.

ROSA, L. P.; HOFFMAN, C. A. A. Cenários sobre o Impacto da Privatização do Setor Elétrico nas Tarifas - O Caso Escelsa. Revista Brasileira de Energia, vol. 4, n. 2, 1995.

SAMPAIO, L. M. B.; RAMOS, F. S.; SAMPAIO, Y. Privatização e Eficiência das Usinas Elétricas Brasileiras. Economia Aplicada, 9(3), jul/set 2005, p. 465-480.

TOLEDO, G. L.; OVALLE, I. I. Estatística básica. 2. ed. São Paulo: Atlas, 1992. 


\section{DADOS DOS AUTORES:}

\section{Solange Garcia}

Universidade de São Paulo, Faculdade de Economia, Administração e Contabilidade de Ribeirão Preto.

Av. Bandeirantes 3900 - Monte Alegre - CEP 14040-900

Monte Alegre

Ribeirão Preto, SP - Brasil

$14040-900$

\section{Paulo Roberto Barbosa Lustosa}

Universidade de Brasília, CCA - Departamento de Ciências Contábeis e Atuariais.

Campus Universitário Darcy Ribeiro

Asa Norte

Brasília, DF - Brasil

70910-900

\section{Nara Rosa Barros}

Universidade de Brasília, CCA - Departamento de Ciências Contábeis e Atuariais.

Campus Universitário Darcy Ribeiro

Asa Norte

Brasília, DF - Brasil

70910-900 